\title{
Welchen Einfluss hat internationale Mobilität auf wissenschaftliche Karrieren?
}

\author{
Ein Forschungsüberblick
}

\section{ZUSAMMENFASSUNG}

In einem systematischen Review werten Nicolai Netz, Svenja Hampel und Valeria Aman 96 empirische Studien zum Einfluss internationaler Mobilität auf wissenschaftliche Karrieren aus. Die Analyse dieses noch jungen, aber in den letzten Jahren sehr dynamischen Forschungsfeldes zeigt, dass internationale Mobilität zum Ausbau wissenschaftlicher Netzwerke beiträgt. Zudem gibt es Hinweise, dass internationale Mobilität die wissenschaftliche Produktivität und Rezeption, das heißt die Anzahl der Publikationen und Zitationen, erhöhen kann. Auch den beruflichen Aufstieg kann internationale Mobilität begünstigen. Sie scheint jedoch ebenso mit durchschnittlich längeren befristeten Beschäftigungsverhältnissen einherzugehen. Andere Karrieredimensionen - wie die Kompetenz- und Persönlichkeitsentwicklung, das wissenschaftliche Fachwissen, der Zugang zu Forschungsinfrastruktur und -finanzierung sowie Reputationsgewinne - wurden erst deutlich seltener und auf Basis weniger belastbarer Forschungsdesigns untersucht.

\section{Einleitung}

Durch Internationalisierungs- und Spezialisierungsprozesse sind Wissenschaftlerinnen und Wissenschaftler zunehmend mit der Option beziehungsweise Notwendigkeit internationaler Mobilität konfrontiert. Aus Sicht der Wissenschaftspolitik stellt internationale Mobilität eine Strategie zur Förderung der Produktion und Diffusion von Wissen dar. Aus individueller Sicht gilt sie als Strategie zur Verbesserung der Karrierechancen. Nicht zuletzt vor dem Hintergrund der erheblichen öffentlichen Investitionen zur Förderung internationaler Wissenschaftlermobilität und des Aufwands für die mobilen Wissenschaftlerinnen und Wissenschaftler stellt sich die Frage, inwiefern internationale Mobilität wissenschaftliche Karrieren tatsächlich beeinflusst.

Dies haben bereits zahlreiche Studien untersucht. Das Forschungsfeld zum Einfluss internationaler Mobilität auf wissenschaftliche Karrieren ist jedoch stark fragmentiert, weil die Diskussionen bislang überwiegend innerhalb bestimmter akademischer

\section{AUTOR}

Dr. Nicolai Netz ist wissenschaftlicher Mitarbeiter am Deutschen Zentrum für Hochschul- und Wissenschaftsforschung (DZHW) und leitet dort die Nachwuchsgruppe „Mobilität von Hochqualifizierten“. 
Disziplinen, Forschungsfelder und Fachzeitschriften verlaufen. Vor diesem Hintergrund versucht ein umfassender Reviewartikel, die existierenden empirischen Studien zum Einfluss internationaler Mobilität auf wissenschaftliche Karrieren zu systematisieren und Ansatzpunkte für zukünftige Forschung zu identifizieren (Netz, Hampel \& Aman, 2020). Ziel des vorliegenden Textes ist es, den Ansatz sowie die Kernergebnisse und Implikationen dieses systematischen Reviews zusammenzufassen.

\section{Methodisches Vorgehen}

Das Review untersucht Wissenschaftlerinnen und Wissenschaftler, die im öffentlichen oder privatwirtschaftlichen Bereich im Rahmen von Forschung und/ oder Lehre tätig sind. Im Fokus steht deren räumliche Mobilität über nationale Grenzen. Dabei werden sowohl zeitweilige Auslandsaufenthalte als auch Formen längerfristig angelegter internationaler Migration berücksichtigt. Das Kerninteresse ist der Einfluss dieser Formen internationaler Mobilität auf die Karrieren von Wissenschaftlerinnen und Wissenschaftlern.

In den Auswertungsprozess wurden nur englischsprachige Studien eingeschlossen, welche die skizzierten Definitionen verwenden und neue empirische Ergebnisse präsentieren. Zunächst wurden dabei alle identifizierten Studien unabhängig von ihrem Design, der methodischen Herangehensweise und Qualität sowie dem Publikationsmedium berücksichtigt. Im Rahmen des Auswertungsprozesses wurde jedoch den multivariaten Studien mit kontrollierten Designs (d.h. statistischen Zusammenhangsanalysen unter Einbezug von mehreren Variablen sowie von mobilen und nicht-mobilen Wissenschaftlerinnen und Wissenschaftlern) größeres Gewicht gegeben, weil diese am besten zur Schätzung kausaler Effekte geeignet sind.

Über verschiedene Kanäle wurde nach Studien gesucht, welche die skizzierten Kriterien erfüllen. Erstens wurde auf Basis eines schrittweise erweiterten Schlagwortkatalogs eine umfassende Recherche in der Scopus-Datenbank durchgeführt. Zweitens wurden Studien einbezogen, die den Autorinnen und dem Autor aufgrund vorheriger Forschungsarbeiten bekannt waren. In den als relevant klassifizierten Studien wurden drittens die Literaturangaben daraufhin begutachtet, ob sie weitere relevante Quellen enthalten. Viertens wurde überprüft, ob sich unter den Studien, welche die bereits identifizierten Publikationen laut Google Scholar zitieren, weitere einschlägige Studien befinden. Über diese vier Kanäle wurden schlussendlich 96 relevante Studien gefunden und in die Auswertung einbezogen.

\section{Zentrale Befunde}

Gemessen am Publikationsjahr der identifizierten Studien ist das Forschungsfeld zum Einfluss internationaler Mobilität auf wissenschaftliche Karrieren noch jung. Vor allem in den letzten Jahren hat es sich jedoch sehr dynamisch entwickelt. Die erste relevante Studie wurde im Jahr 1994 publiziert. 90 Prozent aller relevanten Studien wurden nach 2007 veröffentlicht (Abbildung 1).

\section{ABBILDUNG 1: ANZAHL EINSCHLÄGIGER STUDIEN NACH PUBLIKATIONSJAHR}

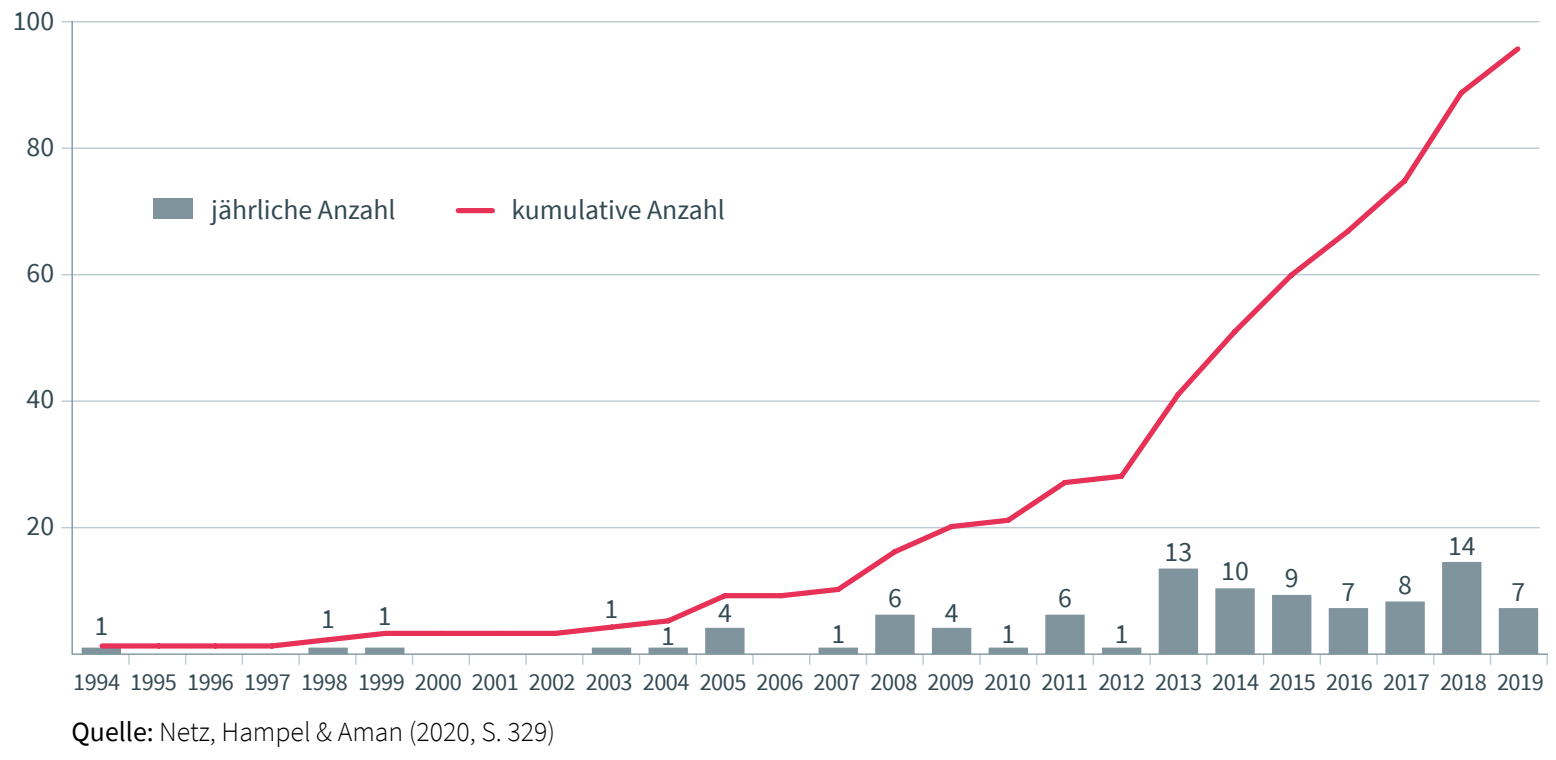


Im Zusammenhang mit den zumeist verwendeten Datenquellen (Publikationsdatenbanken und Befragungen) untersuchen die identifizierten Studien vorrangig publizierende, im akademischen Bereich tätige Wissenschaftlerinnen und Wissenschaftler.

Am häufigsten befassen sich die identifizierten Studien mit Auslandsaufenthalten von mindestens drei Monaten (35 Studien). Seltener untersuchen sie Promotionsphasen im Ausland (16), aktuell im Ausland arbeitende Wissenschaftlerinnen und Wissenschaftler (14), Postdoc-Phasen im Ausland (12) und kürzere Auslandsaufenthalte von weniger als drei Monaten (9). Überraschend viele Studien (42) lassen sich in diese Typologie von Auslandsaufenthalten nicht einsortieren, beispielsweise weil sie die Dauer der Mobilität als erklärende Variable nutzen. Zu diesen Studien zählen auch solche, die internationale Mobilität anhand der Teilnahme an spezifischen Stipendienprogrammen oder bibliometrisch messen - das heißt auf Basis von publizierten Angaben zu den Insti-
Die meisten Studien untersuchen in Europa tätige Wissenschaftlerinnen und Wissenschaftler. Am häufigsten werden Wissenschaftlerinnen und Wissenschaftler analysiert, die in Deutschland (23 Studien), Spanien (21) oder dem Vereinigten Königreich (20) arbeiten. Vergleichsweise selten stehen Wissenschaftlerinnen und Wissenschaftler in den USA (9) im Fokus. Nicht wenige Studien (15) treffen keine Aussage zum Beschäftigungsland der untersuchten Personen, weil sie beispielsweise Wissenschaftlerinnen und Wissenschaftler weltweit bibliometrisch untersuchen.

Mit Abstand am häufigsten analysieren die identifizierten Studien den Einfluss internationaler Mobilität auf wissenschaftliche Netzwerke (47 Studien) und die wissenschaftliche Produktivität (34), also die Anzahl der Publikationen und Patente. Zahlreiche Studien erforschen außerdem, inwiefern internationale Mobilität die berufliche Stellung beeinflusst (26), wie etwa die berufliche Position, das Gehalt oder die berufliche Zufriedenheit. Etwas seltener wird der Einfluss internationaler Mobilität auf die wissenschaftliche Rezeption (23) analysiert, also auf die Anzahl der Zitationen oder den Impact Factor der Zeitschriften, in denen Wissenschaftlerinnen und Wissenschaftler publizieren. Einige
Studien beschäftigen sich mit der Kompetenz- und Persönlichkeitsentwicklung (13) sowie mit Veränderungen der Wissensbasis (13) infolge internationaler Mobilität. Vergleichsweise selten werden mögliche Einflüsse internationaler Mobilität auf den Zugang zu Forschungsinfrastrukturen und Finanzierungsquellen (8) sowie die Akquise symbolischen Kapitals (8) - also Reputationsgewinne durch Aufenthalte an prestigeträchtigen Institutionen - untersucht.

Nicht nur die Anzahl der Studien, sondern auch zusätzliche netzwerkanalytische Zitationsanalysen verdeutlichen, dass sich die identifizierten Teilforschungsfelder in unterschiedlichen Entwicklungsstadien befinden. Insbesondere innerhalb der Teilforschungsfelder zu wissenschaftlichen Netzwerken und zur wissenschaftlichen Produktivität zitieren sich die identifizierten Studien häufig untereinander. In geringerem Maße trifft dies auch auf Studien zur wissenschaftlichen Rezeption zu. Diese Zitationsbezüge weisen darauf hin, dass Autorinnen und Autoren in diesen Teilforschungsfeldern ihre Arbeiten gegenseitig wahrnehmen und eine gemeinsame Wissensbasis entwickelt haben. In den anderen Teilforschungsfeldern lässt sich dies noch nicht beobachten. Gemessen an der bereits beachtlichen Anzahl vorhandener Studien kann insbesondere das Teilforschungsfeld zur beruflichen Stellung als recht fragmentiert bezeichnet werden.

Auch bezogen auf die inhaltlichen Ergebnisse zeigen sich interessante Unterschiede zwischen den Teilforschungsfeldern (Abbildung 2). Vergleichsweise robust ist der Befund, dass internationale Mobilität - unabhängig von der Dauer - zum Ausbau wissenschaftlicher Netzwerke beiträgt: Wissenschaftlerinnen und Wissenschaftler, die im Ausland waren, haben durchschnittlich mehr Co-Autorinnen und Co-Autoren und pflegen häufiger regelmäßige wissenschaftliche Kontakte ins Ausland. Zwar zeigen sich diese positiven Netzwerkeffekte auch noch nach der Rückkehr ins Heimatland, aber die Häufigkeit und Intensität der Kontakte zu Wissenschaftlerinnen und Wissenschaftlern im Ausland scheinen im Zeitverlauf abzunehmen, sofern nicht erneute Auslandsphasen folgen. Einige Studien verweisen auch auf Schwierigkeiten, die wissenschaftlichen Netzwerke im Heimatland aufrecht zu erhalten, und auf Wiedereingliederungsprobleme in heimische Netzwerke nach langen Auslandsaufenthalten. 


\section{ABBILDUNG 2: ERGEBNISSE DER STUDIEN ZUM EINFLUSS INTERNATIONALER MOBILITÄT AUF WISSENSCHAFTLICHE KARRIEREN}

Wissenschaftliche Netzwerke

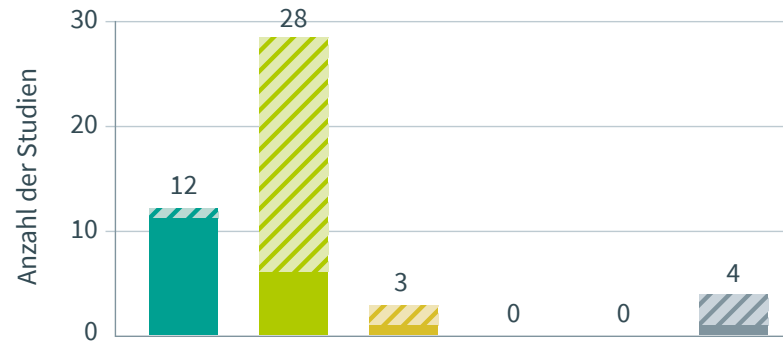

Berufliche Stellung

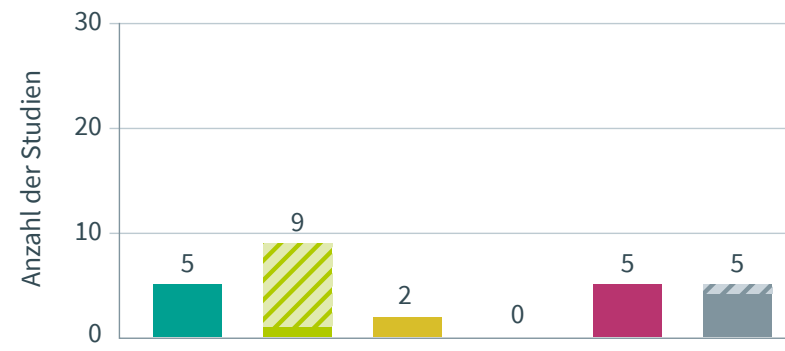

Kompetenz- \& Persönlichkeitsentwicklung

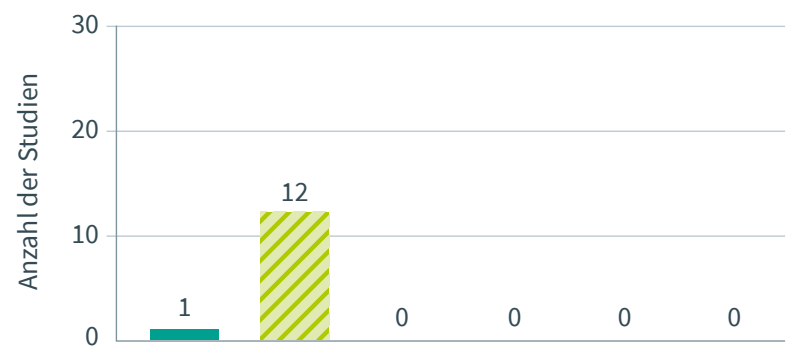

Forschungsinfrastruktur \& -finanzierung

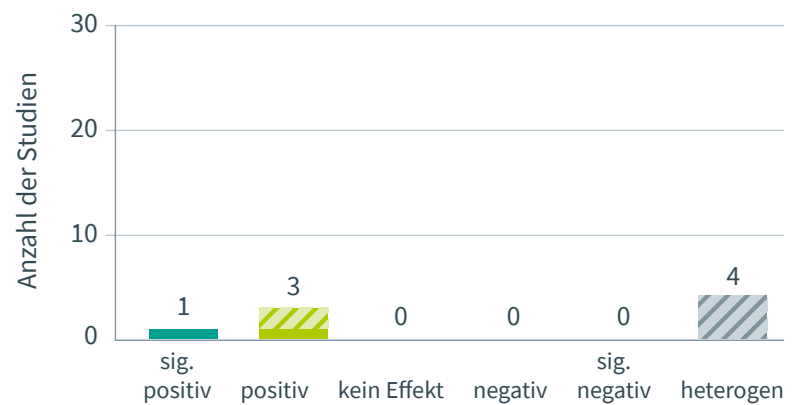

Wissenschaftliche Produktivität

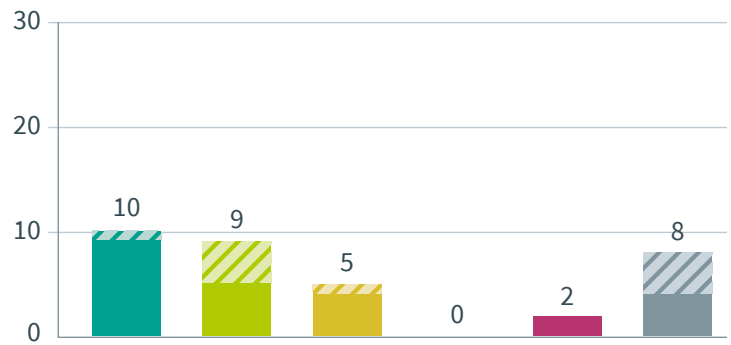

Wissenschaftliche Rezeption

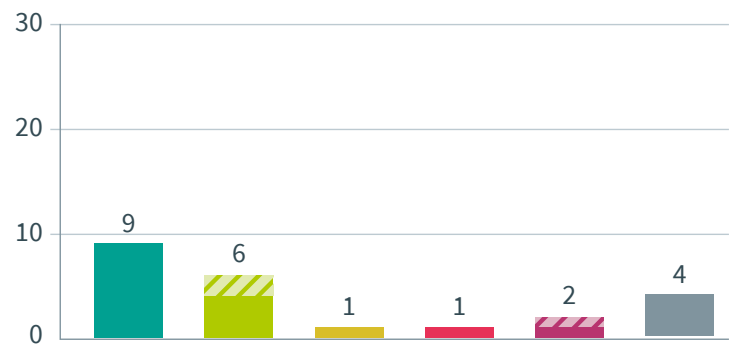

Fachwissen

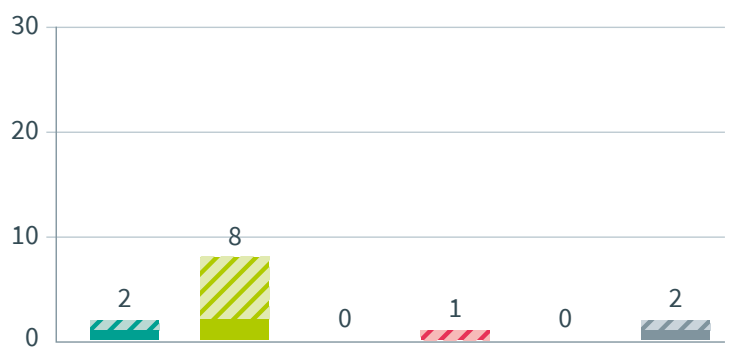

Symbolisches Kapital

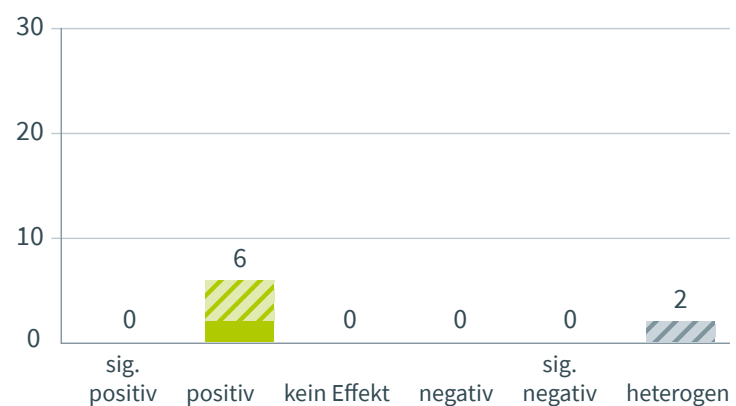

Anmerkung: Die farbig ausgefüllten Balkenteile stehen für Studien, die kontrollierte Designs verwenden (also eine nicht-mobile Kontrollgruppe einbeziehen), während die schraffierten Balkenteile für Studien mit unkontrollierten Designs stehen. Unterschieden werden Studien, die signifikant positive/negative Effekte finden und solche, die Effekte berichten, jedoch keine Signifikanztests vornehmen. Die letztgenannte Kategorie enthält auch Studien, die Signifikanztests beinhalten, aber nicht signifikante positive/negative Effekte berichten. Die Kategorie „kein Effekt" umfasst Studien, die keine beziehungsweise sehr geringe nicht signifikante Effekte finden. Die Kategorie „,heterogen“ umfasst Studien, die unterschiedliche Effekte internationaler Mobilität für unterschiedliche abhängige Variablen (zum Beispiel Publikationen im Vergleich zu Patenten) oder nach dem Beschäftigungsland, der Karrierestufe oder der Disziplin ermitteln. 
Weniger, aber gleichermaßen robuste Analysen stellen positive Effekte internationaler Mobilität auf die wissenschaftliche Produktivität fest. In verschiedenen Studien variieren die Effekte internationaler Mobilität auf die Anzahl der Publikationen und Patente nach Fächergruppen, jedoch bislang ohne klar erkennbares Muster. Positive Produktivitätseffekte scheinen sich vor allem dann einzustellen, wenn ein Wechsel an eine bessere Forschungseinrichtung erfolgt. Anders als im Teilforschungsfeld zu den wissenschaftlichen Netzwerken gibt es auch Studien, die signifikant negative Effekte internationaler Mobilität auf die Produktivität feststellen. Insbesondere in der Zeit nach dem Wechsel an eine Hochschule im Ausland lassen sich übergangsweise Produktivitätseinbußen beobachten, die vermutlich aus der zunächst notwendigen Einarbeitung in neue Aufgaben und Arbeitsumgebungen resultieren.

Im Teilforschungsfeld zur beruflichen Stellung be- re absolute Anzahl an Studien positive Effekte. Die Anzahl der Studien, die signifikant positive Effekte finden, ist hier nur gut halb so hoch wie im zuvor diskutierten Forschungsfeld. Im Einklang mit der Job-Matching-Theorie scheint internationale Mobilität den Zugang zu höheren und besser bezahlten Positionen zu beschleunigen. Es gibt Hinweise, dass dieser Effekt zum Teil dadurch vermittelt wird, dass auslandserfahrene Wissenschaftlerinnen und Wissenschaftler oft mehr Publikationen vorweisen können. Auch wird vermutet, dass internationale Mobilität langfristig zu einer höheren beruflichen Zufriedenheit führen kann. Es gibt aber auch Hinweise, dass auslandserfahrene Wissenschaftlerinnen und Wissenschaftler länger brauchen, bis sie eine unbefristete Position bekommen.

Ähnlich wie im Fall der Netzwerke und der Produktivität finden sich im Teilforschungsfeld zur wissenschaftlichen Rezeption viele - und auch anteilig vergleichsweise häufig - belastbare Studien, die auf positive Effekte verweisen. Vornehmlich auf Basis bibliometrischer Daten zeigen diese Studien, dass auslandserfahrene Wissenschaftlerinnen und Wissenschaftler häufiger zitiert werden und in höher gerankten Zeitschriften publizieren als nicht Auslandserfahrene. Auch diese Effekte scheinen jedoch mit wachsendem zeitlichem Abstand zur Auslandsmobilität abzunehmen.

Weniger und überwiegend deskriptive Studien legen nahe, dass internationale Mobilität Wissenschaftlerinnen und Wissenschaftlern helfen kann, ihre Sprachkenntnisse sowie ihre technischen und methodischen Kompetenzen, ihre Orientierung in anderen Wissenschaftssystemen und ihre Fähigkeit zur Arbeit in diversen Teams zu verbessern. Einige Autorinnen und Autoren vermuten zudem, dass internationale Mobilität das Arbeitsengagement und die Persönlichkeitsentwicklung positiv beeinflussen kann. Anders als in den vorgenannten Fällen sind die Analysen im Teilforschungsfeld zur Kompetenzund Persönlichkeitsentwicklung jedoch insgesamt noch wenig belastbar.

Ähnlich viele und ebenfalls vornehmlich deskriptive Studien liefern Hinweise, dass - insbesondere längerfristige - Auslandsaufenthalte dazu beitragen, dass Wissenschaftlerinnen und Wissenschaftler neues Fachwissen erwerben und ihrerseits Wissens- und Technologietransfer betreiben. In diesem Kontext wird jedoch auch auf Schwierigkeiten verwiesen, das im Ausland erworbene Wissen nach einer Rückkehr ins Heimatland anzuwenden, beispielsweise aufgrund mangelnder technischer Ausstattung oder abweichender Forschungsinteressen an der Heimatinstitution.

Einige wenige Studien argumentieren, dass internationale Mobilität den Zugang zu Forschungsinfrastrukturen und -finanzierung verbessert. Auslandserfahrene Wissenschaftlerinnen und Wissenschaftler scheinen sowohl während ihrer Zeit im Ausland als auch nach ihrer Rückkehr ins Heimatland mit größerem Erfolg Drittmittel einzuwerben. Dieser Befund deckt sich mit der Praxis vieler Forschungsförderorganisationen, internationale Mobilität als ein Signal für die Eignung für eine wissenschaftliche Karriere zu werten.

Erste Studien zum Einfluss internationaler Mobilität auf das symbolische Kapital bestätigen diese Einschätzung. Diese Studien argumentieren, dass internationale Mobilität die Reputation von Wissenschaftlerinnen und Wissenschaftlern erhöhen kann. Maßgeblich scheinen jedoch das Prestige 
der Gastinstitution und das Land der späteren Beschäftigung zu sein. So gibt es Hinweise, dass Auslandsaufenthalte vor allem in Ländern mit sehr forschungsstarken Institutionen positiven Signalwert haben.

Zusammenfassend lässt sich festhalten, dass die Mehrheit der ausgewerteten empirischen Studien positive Effekte internationaler Mobilität auf wissenschaftliche Karrieren ermitteln. Hierunter finden sich auch zahlreiche Studien mit belastbaren Designs. Ebensolche Studien verweisen vereinzelt aber auch auf mögliche negative Effekte internationaler Mobilität.

\section{Fazit}

Die vielfältigen Hinweise auf positive Auswirkungen von internationaler Mobilität auf wissenschaftliche Karrieren können als Rechtfertigung für politische Maßnahmen zur Unterstützung von wissenschaft-
Auch im Bereich der einschlägigen Forschung ist weitere Unterstützung von politischer Seite wichtig. Damit sich das noch junge Forschungsfeld weiterentwickeln kann, bedarf es einer Finanzierung von Datenerhebungen, die längsschnittliche und international vergleichende Kausalanalysen zulassen. Idealerweise sollten neue Datenquellen nicht nur differenziert nach Beschäftigungsländern, sondern auch nach den persönlichen und beruflichen Merkmalen von Wissenschaftlerinnen und Wissenschaftlern - wie dem Geschlecht und der sozialen Herkunft, der akademischen Disziplin oder der Karrierestufe - sowie nach Typen und Frequenz internationaler Mobilität belastbare Aussagen zulassen. Die Genese solcher Daten erfordert sowohl eine längerfristige Finanzierung einschlägiger Datenerhebungsprojekte als auch rechtliche Möglichkeiten zur innovativen Kombination von verschiedenen Datenquellen, wie beispielsweise von Befragungs-, CV-, Sozialversicherungs- und bibliometrischen Daten.

Es ist damit zu rechnen, dass das Forschungsfeld zum Einfluss internationaler Mobilität auf wissenschaftliche Karrieren in den kommenden Jahren weiterhin lebhaft bleibt. Dies gilt nicht zuletzt vor dem Hintergrund der Corona-Pandemie. Diese hat die physische internationale Mobilität stark eingeschränkt. Sie hat aber auch die Bedeutung von virtuellen internationalen Kooperationen vergrößert - und damit von Alternativen zur physischen Auslandsmobilität.

\section{QUELLENHINWEIS}

Netz, N., Hampel, S., \& Aman, V. (2020). What effects does international mobility have on scientists' careers? A systematic review. Research Evaluation, 29(3), 327-351. https:// doi.org/10.1093/reseval/rvaa007

Hier könnten politische Maßnahmen ansetzen: Zur Abmilderung der Karriererisiken internationaler wissenschaftlicher Mobilität könnten die Einrichtung von Tenure-Track-Programmen und supranationale Abkommen zur Übertragbarkeit von im Ausland erworbenen Sozialversicherungsansprüchen weiter vorangetrieben werden. Derartige Maßnahmen würden die Planbarkeit von internationalen Karriereverläufen erleichtern.

\section{ZITIERHINWEIS}

Netz, Nicolai (2021). Welchen Einfluss hat internationale Mobilität auf wissenschaftliche Karrieren? Ein Forschungsüberblick. DAAD Forschung kompakt (1. Fassung vom 22.06.2021). https://doi.org/10.46685/DAADStudien.2021.01 


\section{WEITERFÜHRENDE LITERATUR}

Die folgenden Studien verschaffen beispielhaft Einblick in die identifizierten Teilforschungsfelder.

\section{Wissenschaftliche Netzwerke}

Scellato, G., Franzoni, C., \& Stephan, P. (2015). Migrant scientists and international networks. Research Policy, 44(1), 108-120. https://doi.org/10.1016/j.respol.2014.07.014

\section{Wissenschaftliche Produktivität}

Baruffaldi, S., Marino, M., \& Visentin, F. (2020). Money to move: The effect on researchers of an international mobility grant. Research Policy, 49(8), 104077. https://doi. org/10.1016/j.respol.2020.104077

\section{Berufliche Stellung}

Cruz-Castro, L., \& Sanz-Menéndez, L. (2010). Mobility versus job stability: Assessing tenure and productivity outcomes. Research Policy, 39(1), 27-38. https://doi. org/10.1016/j.respol.2009.11.008

\section{Wissenschaftliche Rezeption}

Jonkers, K., \& Cruz-Castro, L. (2013). Research upon return: The effect of international mobility on scientific ties, production and impact. Research Policy, 42(8), 1366-1377. https://doi.org/10.1016/j.respol.2013.05.005

Kompetenz- \& Persönlichkeitsentwicklung Equeter, E., \& Hellemans, C. (2016). Influence of international mobility on the attitudes of researchers. European Review of Applied Psychology, 66(2), 47-55. https://doi. org/10.1016/j.erap.2016.03.001

\section{Fachwissen}

Aman, V. (2018). A new bibliometric approach to measure knowledge transfer of internationally mobile scientists. Scientometrics, 117(1), 227-247. https://doi.org/10.1007/ s11192-018-2864-X

\section{Forschungsinfrastruktur \& -finanzierung} Cañibano, C., Otamendi, J., \& Andújar, I. (2008). Measuring and assessing researcher mobility from $\mathrm{CV}$ analysis: The case of the Ramón y Cajal programme in Spain. Research Evaluation, 17(1), 17-31. https://doi. org/10.3152/095820208X292797

\section{Symbolisches Kapital}

Bauder, H., Hannan, C.-A., \& Lujan, O. (2017). International Experience in the Academic Field: Knowledge Production, Symbolic Capital, and Mobility Fetishism. Population, Space and Place, 23(6), 1-13. https://doi.org/10.1002/ psp. 2040

\section{IMPRESSUM}

\section{Herausgeber}

Deutscher Akademischer Austauschdienst e.V.

German Academic Exchange Service

Kennedyallee 50, D - 53175 Bonn

www.daad.de/de/impressum

Referat Forschung und Studien - S15

\section{Ansprechpartner}

Dr. Jan Kercher

kercher@daad.de

\section{Juni 2021}

Als digitale Publikation im Internet veröffentlicht

1. Fassung vom 22.06.2021

(c) DAAD - Alle Rechte vorbehalten

Dieses Werk steht unter der Creative Commons Namensnennung - Nicht kommerziell - Weitergabe unter gleichen Bedingungen 3.0 Deutschland Lizenz (CC BY-NC-SA 3.0 DE) https://creativecommons.org/licenses/by-nc-sa/3.0/de/ 